\title{
Correction: ATRIP protects progenitor cells against DNA damage in vivo
}

Gabriel E. Matos-Rodrigues, Paulius Grigaravicius, Bernard S. Lopez, Thomas G. Hofmann, Pierre-Olivier Frappart and Rodrigo A. P. Martins

\section{Correction to: Cell Death and Disease} https://doi.org/10.1038/s41419-020-03090-9 published online 28 October 2020

The original version of this Article contained an error in the name of author Thomas G. Hofmann, which was incorrectly given as Thomas Hofmann.
This has now been corrected in both the PDF and HTML versions of the Article.

Published online: 15 December 2020 